\title{
Reflexiones en torno a la Revista Quaderns del Institut Català d'Antropologia
}

\author{
Consejo de Redacción de Quaderns. ICA. Barcelona
}

\section{RESUMEN}

La revista Quaderns (1. ${ }^{a}$ etapa, 1980-87; 2. ${ }^{\text {a }}$, desde 1994) nació como medio de expresión del Institut Català d'Antropologia. Aunque manteniendo las investigaciones sobre etnología tradicional catalana, esta revista muestra un creciente interés por el tema de las identidades y las fronteras, buscando la comprensión de las sociedades desde la interrelación y apostando por la diversidad, no sólo en el ámbito catalán, ibérico y europeo, sino también en América Latina y el Magreb.

Palabras clave: Antropología social y cultural, Cataluña, Identidades, Fronteras, Diversidad.

\section{SUMMARY}

The journal Quaderns ( $1^{\text {st }}$ period, 1980-87; $2^{\text {nd }}$, since 1994) appeared as the organ of the Catalan Institute of Anthropology. Though dedicated since its first issue to Catalan traditional ethnology, Quaderns reveals an increasing interest in identities and frontiers, aimed at understanding societies from the interrelations affecting them and concerned with diversity in the Catalan, Iberian and European context as well as in that of Latin America and Northwestern Africa.

Key Words: Social and Cultural Anthropology, Catalonia, Identities, Frontiers, Diversity.

Quaderns es una revista promovida por el Institut Català d'Antropologia (ICA) y financiada exclusivamente por sus miembros, cerca de 400 , a quienes va dirigida la mayor parte de la tirada actual, 600 ejemplares, además de a las suscripciones y al intercambio con otras publicaciones. Su nacimiento en mayo de 1980 es el resultado de un proyecto más antiguo que se hace realidad poco después de la constitución oficial del ICA en 1979. Hasta entonces se consideraba la revista como una necesidad en el pa-

RDTP, LVII, 1 (2002): 109-120 
norama de la incipiente antropología en Cataluña y como medio de expresión de unas aproximaciones teóricas y temáticas que reflejasen con fidelidad las investigaciones y los intereses de los fundadores del Institut Català d'Antropologia.

En las páginas que siguen vamos a presentar en primer lugar la trayectoria de la revista y un breve análisis del contenido de los números publicados hasta el momento. Queremos, así mismo, aprovechar el espacio que se nos brinda para reflexionar sobre algunos factores que han impedido que la revista sea expresión fiel y exacta de la actividad y de los intereses científicos de la antropología catalana; mostrar el contraste que supone constatar la vitalidad del ICA, manifestada por medio de sus constantes actividades, como la organización de cursos, conferencias, el número de socios... y las escasas aportaciones de sus miembros a Quaderns, lo que resulta más sorprendente teniendo en cuenta la relevancia profesional y la productividad general de un número notable de sus asociados.

\section{LA REVISTA}

La primera etapa de la revista Quaderns comprende la publicación de seis números editados entre 1980 y 1987. A pesar de que uno de los objetivos principales propuestos por los miembros fundadores del ICA era la publicación de dos números anuales, se demuestra en la práctica que entre 1982 y 1983 no se cumple y en los años 1984 y 1987 solamente se consigue publicar un número anual.

Algunos miembros fundadores, que formaban parte del consejo de redacción, consideraron que era necesario involucrar a nuevos socios y decidieron llevar a la asamblea general del Institut la propuesta de elegir en ella a los miembros de dicho consejo. Esta relación entre miembros fundadores y consejo de redacción queda reflejada en el hecho de que en los dos primeros números, publicados en el mismo año, se incluyan artículos suyos (excepto el de Bernstein en el $n .^{\circ} 2$ ), mientras que a partir del volumen que contiene los números 3 y 4 comienzan a publicarse, de modo mayoritario, trabajos de otros antropólogos nacionales (Josepa Cucó, Isidoro Moreno) e internacionales (Marie, Assier-Andrieu, Pitt-Rivers, Brandes, FabreVassas, Luconi de Borella). La mayor apertura a trabajos de otros profesionales está vinculada también a un interés por ampliar el debate antropológico y la difusión de la revista en el ámbito científico nacional.

El idioma principal utilizado en la revista en los artículos originales y traducciones ha sido el catalán, estructurándose la revista en "Artículos", 


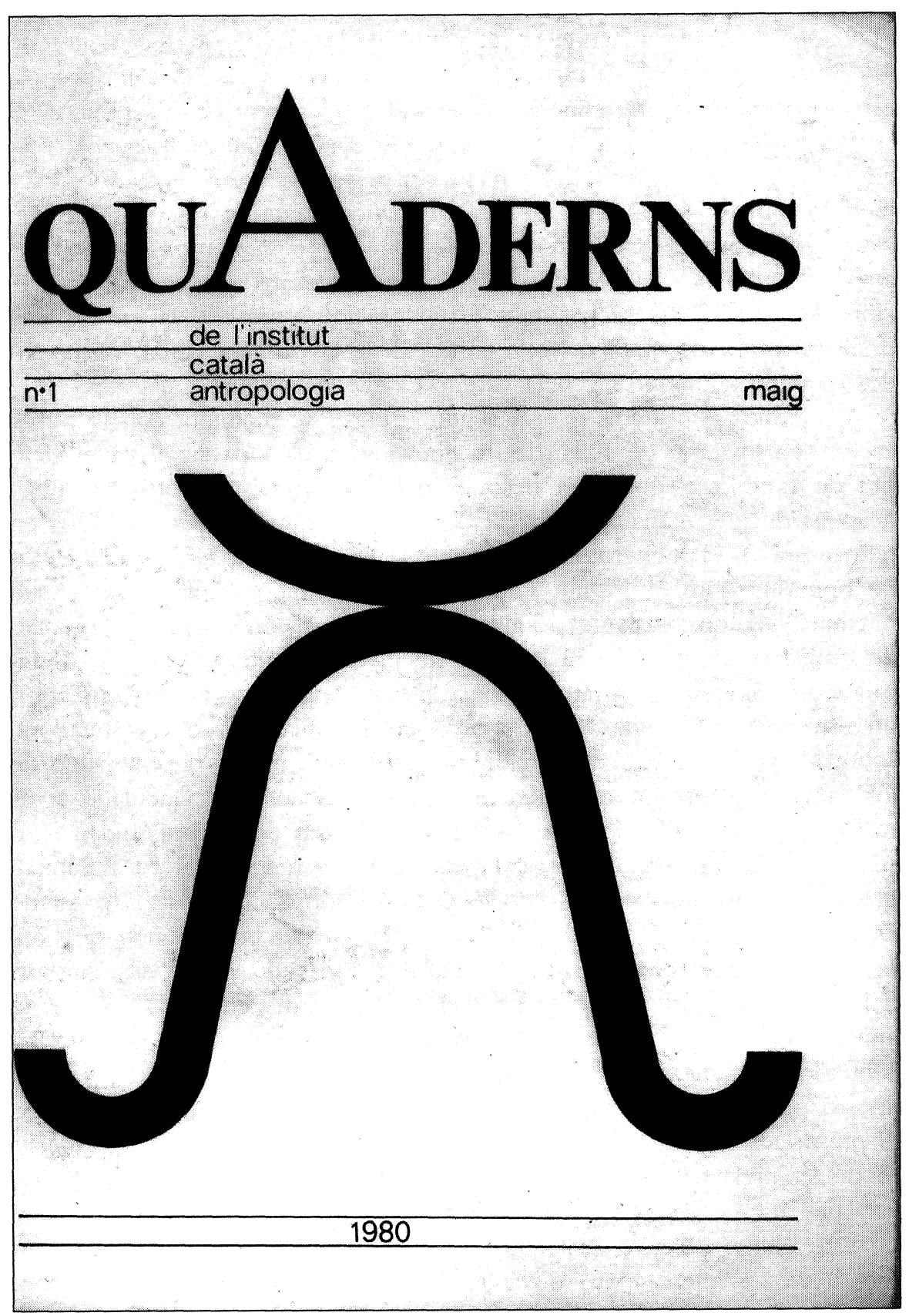


"Críticas y comentarios", "Recensiones" y "Notas". En los números 1 y 2 se incorporó una sección de homenaje a Josep M. Batista y Roca y otra de obituario, dedicada a la figura de Ángel Palerm. Los temas centrales de los artículos giraban en torno a problemas clásicos de la antropología española como el campesinado, la identidad, el parentesco, la política, el simbolismo, la historia y la economía (ver cuadro). Mientras que la sección de críticas y comentarios incluía aspectos metodológicos (objeto y método, aproximación bibliográfica), temáticos (cultura regional, familia, etnicidad, teoría social, cultura e ideología) y comentarios a las obras antropológicas de Alan Marcfarlane y Ángel Palerm (ver cuadro). La sección dedicada a las recensiones estuvo mejor representada en los dos primeros números que en los restantes de esta primera etapa, observándose en los números 5 y 6 la carencia de esta sección.

Podríamos considerar que la segunda etapa se inaugura con la publicación, en invierno de 1994, de un nuevo número 1 para reflejar el espíritu de cambio, y que salía después del número 6, publicado en 1987, después de una etapa de publicación bastante irregular (seis números en quince años). El número 1 de la segunda etapa incluía diversos artículos de opinión sobre el Institut Català d'Antropologia y la antropología en Cataluña, algunos bastante pesimistas, pero otros con una clara voluntad de progreso. Siguiendo esta línea, se incluyeron contribuciones de destacados profesionales de la antropología internacional como James Fernández, un estudio de Yolanda Aixelà, por aquel entonces joven investigadora, sobre los países islámicos y un dossier sobre violencia y deporte, uno de los campos de interés a comienzos de los años noventa, vinculado a los recientes Juegos Olímpicos de Barcelona. El inicio de la continuidad tuvo sus frutos. De ese debate surgió la necesidad de renovación generacional, que se reflejó en un nuevo consejo de redacción de la revista a partir de la siguiente asamblea. Se decidió activar la revista, entendiendo que era necesario recoger el esfuerzo y la tradición presentes desde el comienzo del ICA. El número siguiente apareció en la primavera de 1996 con la antigua numeración $8 / 9$ y desde entonces el nuevo consejo editor se ha centrado en mantener la continuidad de su publicación. El nuevo equipo apostó por la regularidad (aparición de dos números anuales o, cuando el presupuesto no lo permitiera, de un número doble anual) y el aumento de su presencia en la sociedad, tanto en la catalana como en la no catalana, en la segunda etapa.

Este último objetivo se ha manifestado claramente, no sólo en el aumento de los intercambios de la revista, procedentes del Estado español e internacionales, de los que hablaremos a continuación, sino también en la mayor diversidad de los colaboradores y de las temáticas de los artícu- 
los. Así, se constata un aumento de colaboradores en cada número, en la medida en que se ha ampliado el volumen de los ejemplares de la revista, en el número de páginas y en el tamaño del formato, así como también una mayor diversidad interna, aumentando las secciones para conseguir captar un abanico más extenso, que permita recoger los intereses de la antropología en nuestra sociedad. Además de los artículos (en la sección "Elementos", donde se publican los artículos de fondo), base de la revista, y las recensiones (en la sección "Libros"), hemos incorporado las secciones: "Apuntes Etnográficos", "Entrevistas" y "Notas y Comentarios", que sustituyen a las anteriores de la primera época: "Comentarios Críticos" y las "Notas", éstas últimas concentradas, ahora, en el "Full informatiu" del ICA que aparece mensualmente. Las tres secciones actuales han permitido incorporar investigaciones en curso, trabajos descriptivos de interés por su temática, la opinión de autores destacados, a menudo extranjeros de visita en nuestro país como George Foster, Arturo Escobar y Alanis Obomsawin -de los cuales hemos podido obtener las impresiones sobre su trayectoria y contextualizarla en el marco de las corrientes antropológicas contemporáneas-, y artículos de formato más pequeño; estados de la cuestión de ámbitos nuevos o especiales de la disciplina, o bien comentarios sobre obras que comparten una misma temática, e informes sobre investigaciones. También destacamos la diversidad de colaboradores, en la medida en que la apertura de la revista fuera del ámbito catalán ha facilitado su conocimiento en el exterior y el interés de nuevos autores por publicar en ella. Dejando a un lado la presencia de antropólogos de Cataluña, se incluyen los trabajos de otros del Estado español, europeos y latinoamericanos. La apertura se ha centrado también en la participación de autores jóvenes que ha ofrecido nuevas perspectivas, tanto en el ámbito temático, como teórico. Los números 8-9, parte del 12, 13-14, y 15-16 son un ejemplo en este sentido.

En cuanto a las temáticas, la nueva época de Quaderns muestra una ampliación de los intereses generales, aunque se continúa dedicando un espacio importante a los temas clásicos, como las investigaciones sobre etnología tradicional en Cataluña, el parentesco y la identidad, el arte "primitivo", la religión y el ritual, la concepción de la persona, los principios teóricos de la enfermedad y las reflexiones sobre el método etnográfico. Sin embargo, se observa un interés creciente por los campos relacionados con la identidad, tema que ya estuvo presente en la primera etapa. Estas relaciones han aportado una nueva perspectiva a los campos clásicos, como los de ritual o parentesco antes citados. Esta nueva perspectiva busca la comprensión de las sociedades humanas desde la interrelación, con clara voluntad de superación de la delimitación de los 
viejos campos de forma unívoca y aislada. Esta es una de las grandes líneas que marcan los estudios contemporáneos de la antropología y que ha mostrado nuestra publicación de forma muy clara: la dedicación a la inmigración interna y la denominada "extracomunitaria". Pero este énfasis en las interrelaciones lo encontramos también claramente en temas contemporáneos, como los estudios sobre alteridad y frontera en el Mediterráneo; identidades, fronteras y ciudadanía en los Pirineos; recomposición de etnias y fronteras étnicas en África; relaciones de intercambio entre sociedades indígenas y no indígenas en América Latina; modernización en el medio rural mexicano; etc.

En la sección "Elementos", el tema del desarrollo es otro de los intereses recurrentes de la antropología publicada en Quaderns. Dentro de estas nuevas líneas no podemos olvidar tampoco las vinculadas a las técnicas actuales de información etnográfica, como el cine etnográfico, o a cuestiones más propias de la antropología política y la antropología urbana; así como a campos temáticos especializados, como la antropología de la alimentación o la relación entre alimentación y sexualidad.

La sección de "Recensiones" ha tratado de cubrir aquellos ámbitos temáticos y regionales no tratados en los artículos, de manera que en conjunto podemos establecer que, tanto en el terreno de la especialización temática y regional, como en el ámbito teórico, Quaderns ha apostado por la apertura y la diversidad. Se han recogido análisis de obras de diferentes influencias teóricas y tradiciones, como la francesa, la británica y la norteamericana, así como de las más periféricas.

En definitiva, podemos concluir que, tal y como se refleja en la revista Quaderns desde 1995, han predominado algunas áreas temáticas o regionales, sin que se hayan realizado números monográficos. No obstante, en el número 10 han dominado las investigaciones sobre América Latina y en el 11, acerca de la inmigración y la identidad. La antropología sobre América Latina, tanto indígena como no indígena, ha estado presente en la mayoría de los números, hecho que señala la atracción que esta área regional tiene para la antropología contemporánea de nuestro país, del mismo modo que existe un interés continuado por la temática sobre inmigración. Igualmente, observamos una presencia recurrente de cuestiones vinculadas con el Magreb, tanto por lo que respecta a la inmigración, como por los aspectos etnográficos de las sociedades de origen. 


\begin{tabular}{|c|c|c|c|c|c|c|c|}
\hline AÑo & NOMERO & EPOCA & ESTRUCTURA & $\begin{array}{c}\text { IDIOMA } \\
\text { (Articulos) }\end{array}$ & TEMÁTICA (Articulos) & $\begin{array}{l}\text { TEMÁTICA (Críticas y } \\
\text { comentarios) }\end{array}$ & AREA \\
\hline 1980 & 1 & Mayo & \begin{tabular}{|c|} 
Articulos, criticas y \\
connentarios, recensiones \\
y homenaje \\
\end{tabular} & Ciltalín & $\begin{array}{l}\text { Identidad, historia de la } \\
\text { antropología, parentesco, } \\
\text { politica }\end{array}$ & $\begin{array}{l}\text { Temáticos rcultura } \\
\text { regional), critica a obra } \\
\text { antropológica }\end{array}$ & Paises cattalines \\
\hline 1980 & 2 & Noviembre & $\begin{array}{c}\text { Articulos, bibliografia, } \\
\text { críticas y comentarios, } \\
\text { obituario, recensiones, } \\
\text { notis }\end{array}$ & $\begin{array}{l}\text { Cattalín, } \\
\text { castellano }\end{array}$ & $\begin{array}{c}\text { Parentesco, teoria antropológica, } \\
\text { simlrolismo, rituales }\end{array}$ & $\begin{array}{l}\text { Metoxdoligicicos } \\
\text { (aprosimación } \\
\text { bilblicgrafifica) }\end{array}$ & $\begin{array}{l}\text { Pailises cattalanes, Penínsulit } \\
\text { Ilbérica }\end{array}$ \\
\hline 1981 & $3 / 4$ & $\begin{array}{c}\text { Mayo } \\
\text { noviembre }\end{array}$ & $\begin{array}{c}\text { Articulos, críticas y } \\
\text { comentarios, recensiones } \\
\text { y notass } \\
\end{array}$ & Caltalán & $\begin{array}{l}\text { Ecconomial, identidad. } \\
\text { cimpesinado, historiat }\end{array}$ & 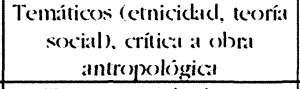 & $\begin{array}{l}\text { Palises citalianes, Penínsulit } \\
\text { Ilxérica, Francia }\end{array}$ \\
\hline 1984 & 5 & & $\begin{array}{c}\text { Articulos, reflexiones y } \\
\text { comentarios, notas } \\
\text { etnogrificas }\end{array}$ & Cittalín & $\begin{array}{l}\text { Jurídica, campesinado, } \\
\text { parentesco. historia de la } \\
\text { intropología, politica }\end{array}$ & 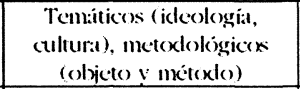 & $\begin{array}{l}\text { Palises catalanes, Penínsulat } \\
\text { Ilbérical }\end{array}$ \\
\hline 1987 & 6 & - & Articulos & Cittalín & $\begin{array}{c}\text { Parentesco, politica, simbolismo, } \\
\text { rituakes, psiquiatria }\end{array}$ & - & $\begin{array}{c}\text { Paises citalanes, Francia, } \\
\text { Méxicos }\end{array}$ \\
\hline 1994 & 7 & Invierno & $\begin{array}{l}\text { Discusion, elementos, } \\
\text { dossier, libros y noticas }\end{array}$ & Catalín & $\begin{array}{c}\text { Teoría : intropoltigica, cambio } \\
\text { social, depone }\end{array}$ & 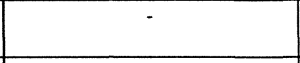 & Países islämicus \\
\hline $1905 / 6$ & $8 / 9$ & Primatveta & $\begin{array}{c}\text { Elementos, librcs, noticias, } \\
\text { comentarios y notas }\end{array}$ & $\begin{array}{l}\text { Catalain, } \\
\text { castellano }\end{array}$ & $\begin{array}{l}\text { Simbolismo, enfermediad, } \\
\text { religión, metoclología, } \\
\text { :limentación }\end{array}$ & 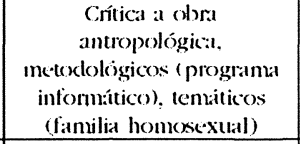 & $\begin{array}{l}\text { Africa Occidental, Paíises } \\
\text { Istámicos. Irtandi. }\end{array}$ \\
\hline 1997 & 10 & Invierno & $\begin{array}{c}\text { Elementos, librox, noticias, } \\
\text { comentarios y notas de } \\
\text { lectura }\end{array}$ & $\begin{array}{l}\text { Inglés, } \\
\text { castellano }\end{array}$ & $\begin{array}{l}\text { Econonía, rituales, religion, } \\
\text { identidad, visual }\end{array}$ & $\begin{array}{l}\text { Tenníticos (faumiliat c } \\
\text { historia secial) }\end{array}$ & Latinoamérica \\
\hline 1997 & 11 & Otoño & Elementos, libros & $\begin{array}{c}\begin{array}{c}\text { Castellano, } \\
\text { inglés, } \\
\text { caltalan }\end{array} \\
\end{array}$ & Inmigracion, Identidad & - & $\begin{array}{c}\text { Península Ibérica, Países } \\
\text { Cattalanes }\end{array}$ \\
\hline 1998 & 12 & Invierno & $\begin{array}{l}\text { Elementos, entrevista y } \\
\text { libros }\end{array}$ & $\begin{array}{l}\text { Inglés, } \\
\text { catalin, } \\
\text { castellano }\end{array}$ & $\begin{array}{l}\text { Religión, ante, historia de ba } \\
\text { antropologia, metrolologia, } \\
\text { economia }\end{array}$ & - & Países Cattilanes \\
\hline 1999$)(01$ & $13 / 14$ & Invierno & $\begin{array}{l}\text { Elementos, entrevista, } \\
\text { apuntes einogríficos, } \\
\text { libros } \\
\end{array}$ & $\begin{array}{l}\text { Catalán, } \\
\text { castellano, } \\
\text { francés } \\
\end{array}$ & $\begin{array}{l}\text { Religión, inmigración, urlyana, } \\
\text { políicical }\end{array}$ & $\begin{array}{l}\text { Temhíticos (resistencia } \\
\text { campesin:t, emociones) }\end{array}$ & $\begin{array}{l}\text { P:aises Catalanes. Mauritaniat } \\
\text { Brasil }\end{array}$ \\
\hline $20010 / 1$ & $15 / 16$ & Invierno & $\begin{array}{l}\text { Elementos, entrevista, } \\
\text { apuntes einogrificos, } \\
\text { libros }\end{array}$ & $\begin{array}{l}\text { Catalan, } \\
\text { castellano, } \\
\text { francés }\end{array}$ & $\begin{array}{l}\text { Parentesco, Politica, Religion, } \\
\text { Economia, Psicoanalisis }\end{array}$ & $\begin{array}{l}\text { Temáticos (expetiencia, } \\
\text { personat, atte) }\end{array}$ & $\begin{array}{l}\text { Paises Catalanes, Francia, } \\
\text { Argentina }\end{array}$ \\
\hline
\end{tabular}




\section{UNAS NOTAS AL MARGEN}

No estamos en condiciones de hacer una historia de lo que pueda haber representado el ICA o su revista, Quaderns, para la antropología catalana, aunque parece una tarea ineludible que habrá que abordar en algún momento, para comprender la realidad actual de nuestra disciplina en Cataluña. No obstante la trayectoria de la revista puede ayudarnos a señalar algunos de los factores que impiden, hasta ahora, ser demasiado optimistas; lo que no significa que no deban abrigarse esperanzas de que con la solución de algunos de los problemas que vamos a señalar pueda darse un nuevo impulso a una comunidad de antropólogos radicalmente distinta a la que inició su andadura institucional en la década de 1970, aunque buena parte de sus miembros sean los mismos de entonces.

El problema de la antropología en Cataluña -aunque estamos convencidos de que en esto no podemos presumir de "hecho diferencial»no es que no dispongamos de individualidades que estén por encima de la media de las individualidades en el panorama mundial de la antropología. El problema no es de individuos, sino del colectivo como tal, de la falta de intercambio intelectual y de las dificultades para hacer una obra común; por ejemplo, una revista que agrupe los esfuerzos de las varias que ya existen, para que la antropología catalana pueda tener la presencia e influencia en la comunidad científica internacional y que la dispersión actual impide alcanzar. El ICA como institución y algunos de sus miembros individualmente lo han intentado sin éxito, hasta la fecha. La ausencia de un órgano común es sólo un síntoma del problema más importante, la falta de una comunidad científica que refleje el vigor que, desde otras perspectivas, tiene la antropología en Cataluña.

Las ideas aquí expuestas intentan recoger algunos comentarios de diferentes miembros del ICA, y no reflejan necesariamente las de todos sus socios, ni, tal vez, las de todas las personas del consejo de redacción actual de la revista. Quizá, para exponerlas con profundidad y matices suficientes sería necesario organizar un debate sobre Quaderns entre las distintas generaciones, similar al que tuvo lugar con motivo del decimoquinto aniversario del ICA, recogido en las páginas del primer número de la segunda época. A él remitimos a las personas interesadas.

El vigésimo segundo aniversario de la revista Quaderns coincide con la salida de un volumen doble que contiene los números 17 y 18 . La escasa diferencia entre años de existencia y números publicados debería llenarnos de satisfacción por lo logrado, aunque para cumplir con los objetivos primeros esta entrega debería corresponder al número 44. Aun 
sin haber podido alcanzar ese propósito, deberíamos considerar un logro que la revista siga viva, si tenemos en cuenta las conocidas dificultades por las que han pasado y pasan las publicaciones científicas en estos tiempos, especialmente aquéllas que, por haber querido mantener su independencia, o por no haber interesado a nadie que la perdiese, no han tenido más recursos que los propios. Sin embargo, si atendemos a otras variables además de la mera existencia, el éxito de la revista es más dudoso y la satisfacción, por tanto, relativa. La declaración de intenciones del primer equipo, expuesta en el n. ${ }^{\circ}$, de mayo de 1980, puede ayudar a entenderlo:

La revista "Quaderns de l'Institut Català d'Antropologia" sorgeix com a portaeu de les activitats i treballs individuals i/o col-lectius dels membres de l'ICA i dels seus colllaboradors.

La revista "Quaderns de l'ICA" preten ésser a més un instrument útil per promoure la col-laboració entre els individuus i grups que treballen i investiguen en el terreny de l'Antropologia, en el marc dels Països Catalans.

La revista "Quaderns de l'ICA" vol ésser també un fòrum per a la discusió de temàtiques rellevants, tant per a l'Antropologia com per d'altres disciplines, com per exemple, l'evolució de l'estructura familiar, els sistemes d'herència, la diferenciació dintre de la pagesia, el nacionalisme, etc., per tal de desenvolupar un acostament interdisciplinari entre les mateixes.

La revista "Quaderns de l'ICA" publicará prioritàriamente estudis i treballs referents a l'àmbit dels Països Catalans, sense marginar però les contribuciones significatives referents a altres parts de la Península Ibèrica i, excepcionalment, d'altres àrees.

Salvo quizá en lo que se refiere a los campos temáticos sobre los que discutir, podría afirmarse que todos los equipos que se han sucedido en la redacción de Quaderns, compartirían las intenciones de aquel primer consejo de redacción. Sin embargo, ninguno de los objetivos se cumplió estrictamente en la primera etapa (ver cuadro). Uno de los miembros del primer consejo de redacción nos señalaba las dificultades para conseguir originales como uno de los problemas a los que se había enfrentado la redacción. Algo similar ha pasado en la segunda etapa; aunque no han faltado originales, no siempre ha sido posible ser demasiado estrictos en la selección. Los trabajos rechazados han sido escasísimos, prueba de que todos ellos estaban a un buen nivel, según los criterios del equipo, pero también de que no podíamos ser muy exigentes.

Otro de los incumplimientos, aunque no quede recogido en la declaración anterior, ha sido el de publicar en catalán. En la primera etapa, prácticamente todos los artículos estaban escritos o se traducían al catalán. En la segunda época se manifestó la voluntad de mejorar la difusión 
de la revista, incluso se barajó la posibilidad de publicarla en inglés, pero ante el rechazo de esta idea en la Asamblea del ICA, se decidió publicar en las lenguas en las que llegasen los artículos, las lenguas románicas más comunes e inglés.

Los consejos de redacción han debido asumir todas las tareas de edición, lo que significa leer los originales, recabar colaboraciones para las reseñas de libros — cuando no escribirlas directamente-, corregir los textos, mantener contacto con los autores, etc. De no haber sido por la eficacia y buena disposición de la secretaria del ICA, Araceli Muñoz, nuestra tarea hubiera sido mucho más difícil de realizar. Un inconveniente importante para la difusión de la revista ha sido la distribución, que se intentó paliar en la segunda etapa mediante la coedición con la editorial Icaria; esta colaboración no resolvió el problema de la distribución y las dificultades económicas de la revista se agravaron, por lo que después de varios números, decidimos continuar en solitario.

De cómo han afectado las diversas dificultades con las que se han encontrado los consejos de redacción, pueden ser muestra los párrafos siguientes extraídos del "Editorial" del primer número de la segunda época, de invierno de 1994:

És molt possible que la història d'una revista científica anomenada Quaderns de l'ICA pugui ser pensada a la manera d'espill en què resten, como si hi fossin reflectides, les pròpies condiciones vitals d'aquella entitat de la qual va voler ser portaveu: illlusionada i fràgil; entusiasta i al mateix temps insegura. Sis números en quinze anys no semblen gaire cosa, i el cert és que ni tan sols una capçalera o un format comuns han pogut constatar el batec d'una certa personalitat que nués els continguts ni tampoc certificar l'existència dùn projecte intel-lectual sòlid. Ara, set anys després de la seva darrera entrega, reapareix - encara que potser caldria millor dir que ressuscita- el Quaderns, amb la voluntat d'inaugurar una nova etapa, idèntica, a la fi, a aquella altra que es pretén que inicii el mateix Institut Català d'Antropologia.

Fer una declaració de pretensions implicaria en aquest moment, sens dubte, una imprudència, car, com tants altres propòsits semblats als nostros, les possibilitats de fracàs són massa grans per exaltar ara unes promeses que és fàcil veure decebudes. Direm, tan sols, que hi ha el determini ferm de treballar seriosament i amb rigor perquè l'ICA i aquest òrgan de comunicació que aquí s'obre perdurin, i confiem que aquest esforç sigui la premissa d'una fortuna: contribuir a enfortir i perllongar una tradició científica nacional que ho mereix.

Como es fácil de apreciar, la melancolía que refleja el "Editorial" del primer número de la segunda época, contrasta con la rotunda voluntad con que se expresan los objetivos de la primera. ¿Qué ha ocurrido entre una y otra época que justifique el tono utilizado por la Junta del ICA que retoma la publicación de la revista en 1994? Seguramente lo que en 
el conjunto de la sociedad catalana: Tras la euforia que trajeron los últimos años del franquismo, que lanzó a una generación a participar y a comprometerse, a crear, con grandes dosis de voluntarismo, proyectos propios en aquellos ámbitos que le afectaban, vino la profesionalización para unos pocos y el desencanto para muchos. Nacieron profesionales de la vida política, pero también de otras muchas actividades de la llamada sociedad civil; la antropología y los antropólogos no iban a quedarse al margen de esa tendencia. Paradójicamente, o quizá como consecuencia de ello, que una parte de los antropólogos se profesionalizase, y tal vez otra esperase hacerlo, significó el debilitamiento de aquel primer ímpetu, que tal vez sólo exista como mito de origen para los miembros más recientes del ICA, y para quienes lo vivieron en su juventud, hace ya alrededor de 30 años. Poco a poco las asambleas del ICA fueron vaciándose, aunque no se redujo significativamente el número de socios. Parecía que éstos delegasen en la Junta del ICA la realización de actividades de las que en otro tiempo participaban o eran impulsores. Como había ocurrido en la vida política, se dejó a los profesionales la representación, las decisiones y las actividades $\longrightarrow$ las acaparamos, como reprochan algunos socios. Lo que no significa que sólo los profesionales de la antropología, quienes se ganan la vida como antropólogos, hayan participado en el ICA, o en la revista Quaderns, pero sí que han sido mayoría, al menos en el consejo de redacción de esta última.

El camino del ICA y de su revista van juntos y no extraña, en las condiciones de falta de participación de los socios en las que se desenvuelve el ICA, que las contribuciones a la revista sean también escasas. No obstante, más influencia que todo eso en el estado actual de la revista, y del ICA, tiene la falta de discusión, de debate teórico, de espacios en los que exponer y discutir sobre las investigaciones en marcha o ya realizadas y el que, cuando alguien intenta crear dicho espacio, la participación de los antropólogos sea escasa.

Estas reflexiones no deben inducir a pensar que existe desmoralización por parte del consejo de redacción, sino todo lo contrario, si se tiene en cuenta cómo nos planteamos el futuro inmediato de la revista: Con la idea de conseguir una mayor difusión y participación, además de aligerar la carga económica, casi insoportable, que supone la publicación para el Institut Català d'Antropología, el consejo de redacción llevó a la Junta y ésta a la Asamblea un proyecto que permitirá mejorar -0 eso esperamos- la situación de Quaderns en muchos sentidos. Por un lado, se propone editar una revista electrónica, más accesible a los profesionales, estudiantes y personas interesadas en la antropología que no sean socios del ICA. Al mismo tiempo, confiamos en recibir mayor número de 
artículos y no tener impedimentos para publicar todos los que presenten la calidad necesaria, además de evitar las largas esperas que muchos de los autores soportaban hasta ver publicados sus artículos. Paralelamente, se publicará un número monográfico en papel cada año, cuya coordinación se encargará a especialistas en el tema elegido.

La segunda etapa de la revista no disponía de un comité asesor, aunque en ocasiones algunos especialistas se habían prestado a leer artículos que el consejo de redacción les remitía, por no tener suficiente seguridad para evaluarlos. De cara a la revista electrónica hemos contactado, y recibido respuestas positivas, a distintos antropólogos de Cataluña para que constituyan el comité asesor de la revista, lo que sin duda mejorará la publicación. En esa confianza, invitamos a quienes estén interesados en la revista Quaderns para que, a partir del próximo curso, visiten la página del ICA en la red: $h t t p: / / w w w . u b . e s / i c a$, en la que confiamos puedan encontrar ya el primer número de la revista Quaderns en soporte electrónico. 